CRYSTALLOGRAPHIC COMMUNICATIONS

ISSN 2056-9890

\title{
Crystal structure and Hirshfeld surface analysis of methyl 4-[(E)-2-(5-bromo-2-methoxybenzyl- idene)hydrazinyl]-3-nitrobenzoate
}

\author{
Tanvirbanu J. Malek, ${ }^{a}$ Sahaj A. Gandhi, ${ }^{\mathrm{b} *}$ Vijay Barot, ${ }^{\mathrm{c}}$ Mukesh Patel ${ }^{\mathrm{C}}$ and \\ Urmila H. Patel ${ }^{\mathrm{d}}$
}

Received 9 July 2018

Accepted 8 August 2018

Edited by D. Chopra, Indian Institute of Science Education and Research Bhopal, India

Keywords: crystal structure; hydrazine derivative; graph set motif; hydrogen bond; Hirshfeld surface analysis.

CCDC reference: 1860856

Supporting information: this article has supporting information at journals.iucr.org/e

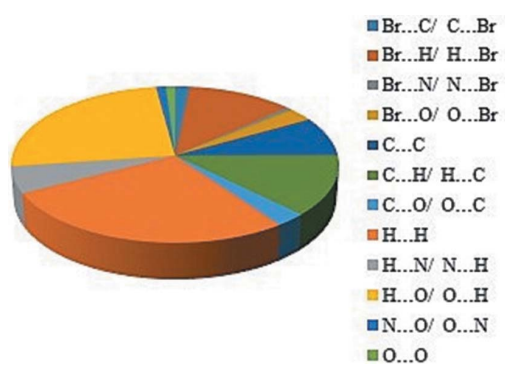

OPEN $\odot$ ACCESS
aLDRP-Institute of Technology \& Research, Kadi Sarva Vishwavidhayalay, Gandhinagar, India, 'bhavan's Shri I. L. Pandya Arts -Science and Smt. J. M. Shah Commerce College, Dakor, Gujarat, India, 'P.G. Center in Chemistry, Smt. S. M. Panchal Science College, Talod, India, and d Department of Physics, Sardar Patel University, Vallabh Vidyanagar, India. *Correspondence e-mail: sahajg7@gmail.com

The title compound, $\mathrm{C}_{16} \mathrm{H}_{14} \mathrm{BrN}_{3} \mathrm{O}_{5}$, is a novel halogen (Br) substituted hydrazine derivative. The hydrazine derivatives were the group of compounds with the general structure, $R_{1} R_{2} \mathrm{C}=\mathrm{NNH}_{2}$ (Uppal et al., 2011), with the central $R \mathrm{C}=\mathrm{NNH}_{2}$ moiety bridging two different groups on both sides. An all-trans configuration of the backbone $\left(R \mathrm{C}=\mathrm{NNH}_{2}\right)$ results in an extended molecular conformation. The dihedral angle between the 5-bromo-2-methoxyphenyl ring and the nitrophenyl ring is $4.4(3)^{\circ}$. Intramolecular $\mathrm{N}-\mathrm{H} \cdots \mathrm{O}$ interactions form $S(6)$ graph-set motifs, while $\mathrm{C}-\mathrm{H} \cdots \mathrm{O}$ and $\mathrm{C}-\mathrm{H} \cdots \mathrm{N}$ interactions form $S(5)$ graph-set motifs. Symmetry-related molecules are linked by $\mathrm{C}-\mathrm{H} \cdots \mathrm{O}$ intermolecular interactions forming an $R_{2}^{1}(10)$ graph-set motif. There are nearly faceto-face directional specific $\pi-\pi$ stacking interactions between the centroids of the nitrophenyl ring and the benzene ring of the 5-bromo-2-methoxy group [centroid-centroid distance $=3.6121(5) \AA$ and slippage $=1.115 \AA$ ] , which also contributes to the molecular packing. The Hirshfeld surface analysis was performed in order to visualize, explore and quantify the intermolecular interactions in the crystal lattice of the title compound.

\section{Chemical context}

Hydrazine and its derivatives have attracted much attention due to their synthetic potential for organic and inorganic chemical reactions and diverse useful properties (Levrand $e t$ al., 2007; Li et al., 2011). Hydrazine-based coupling methods are used in medical biotechnology to couple drugs to targeted antibodies, e.g. antibodies against a certain type of cancer cell (Wu et al., 2005). Hydrazine possesses diverse biological and pharmacological properties, such as antimicrobial, antiinflammatory, analgesic, antifungal, antitubercular, antiviral, anticancer, antiplatelet, antimalarial, anticonvulsant, cardioprotective, antihelmintic, antiprotozoal (Rollas \& Küçükgüzel, 2007), antitrypanosomal and antischistosomiasis (Narang et al., 2012). These compounds contain a $\mathrm{C}=\mathrm{N}$ bond, which is conjugated with a lone pair of electrons of the functional $\mathrm{N}$ atom (Corey \& Enders, 1976). The $\mathrm{N}$ atom of the hydrazine is nucleophilic and the $\mathrm{C}$ atom has both an electrophilic and a nucleophilic nature (Corey \& Enders, 1976). The $\alpha$-hydrogen of hydrazine is more potent than that of acidic ketones (Belskaya et al., 2010). The combination of hydrazine with other functional groups results in new compounds with unique physical and chemical characteristics (Xavier et al., 2012). Owing to their biological and pharmacological properties, 
Table 1

Hydrogen-bond geometry $\left(\AA,^{\circ}\right)$.

\begin{tabular}{|c|c|c|c|c|}
\hline$D-\mathrm{H} \cdots A$ & $D-\mathrm{H}$ & $\mathrm{H} \cdots A$ & $D \cdots A$ & $D-\mathrm{H} \cdots A$ \\
\hline $\mathrm{N} 2-\mathrm{H} 2 A \cdots \mathrm{O} 4$ & 0.83 & 2.03 & $2.635(3)$ & 129 \\
\hline $\mathrm{C} 3-\mathrm{H} 3 \cdots \mathrm{O} 1$ & 0.93 & 2.39 & $2.712(4)$ & 100 \\
\hline $\mathrm{C} 6-\mathrm{H} 6 \cdots \mathrm{N} 3$ & 0.93 & 2.40 & $2.731(4)$ & 101 \\
\hline $\mathrm{C} 6-\mathrm{H} 6 \cdots \mathrm{O} 4^{\mathrm{i}}$ & 0.93 & 2.59 & $3.444(5)$ & 152 \\
\hline $\mathrm{C} 15-\mathrm{H} 15 \cdots \mathrm{O} 4^{\mathrm{i}}$ & 0.93 & 2.46 & $3.358(4)$ & 161 \\
\hline
\end{tabular}

Symmetry code: (i) $x-\frac{1}{2},-y-\frac{1}{2}, z-\frac{1}{2}$.

hydrazine derivatives play an important role for the synthesis of heterocyclic compounds (Banerjee et al., 2009).<smiles>COC(=O)c1ccc(N/N=C/c2cc(Br)ccc2OC)c([N+](=O)[O-])c1</smiles>

\section{Structural commentary}

Fig. 1 displays the title molecule with the atom-labelling scheme. Intramolecular $\mathrm{N} 2-\mathrm{H} 2 A \cdots \mathrm{O} 4$ interactions form $S$ (6) graph-set motifs and $\mathrm{C} 3-\mathrm{H} 3 \cdots \mathrm{O} 1$ and $\mathrm{C} 6-\mathrm{H} 6 \cdots \mathrm{N} 3$ interactions form $S(5)$ graph-set motifs. The central bridging moiety $\mathrm{R}_{2} \mathrm{C}=\mathrm{NNHR}_{1}$ adopts an all-trans conformation about the $\mathrm{C} 10-\mathrm{C} 9, \mathrm{C} 9-\mathrm{N} 3, \mathrm{~N} 3-\mathrm{N} 2$ and $\mathrm{N} 2-\mathrm{C} 5$ bonds, with torsion angles of $176.0(6),-178.1(5),-177.0(6)$ and $173.6(6)^{\circ}$, leading to an extended molecular conformation, thereby causing the terminal bromomethoxyphenyl ring and nitrophenylring to occupy almost the same plane; the dihedral angle between the rings is $4.4(3)^{\circ}$.

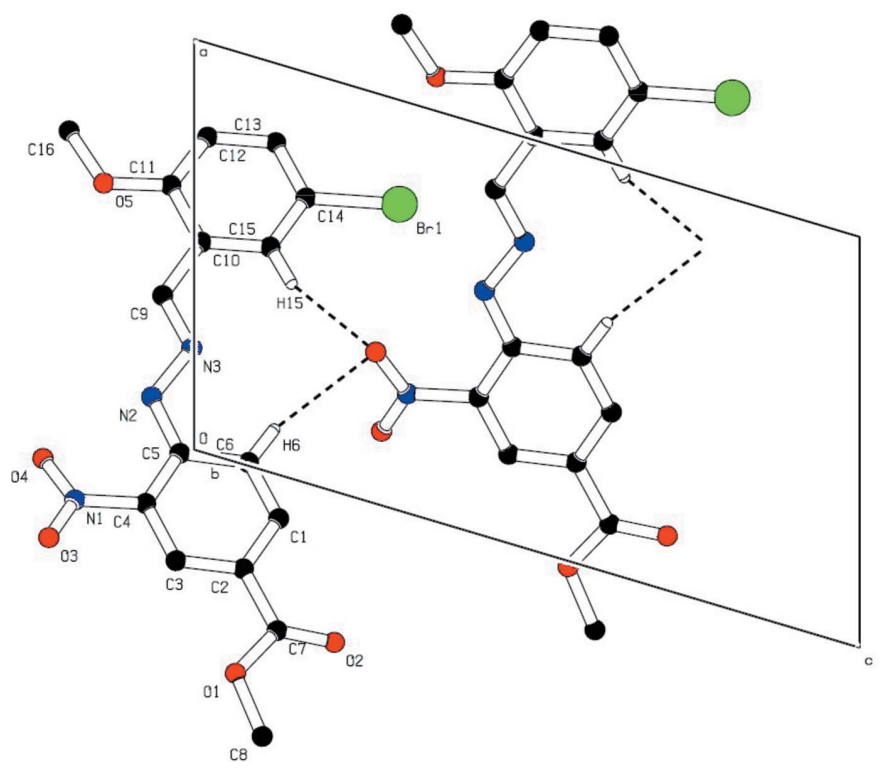

Figure 2

A view of part of the crystal structure of the title compound, showing the formation of $\mathrm{C}-\mathrm{H} \cdots \mathrm{O}$ hydrogen bonds (dashed bonds).

\section{Supramolecular features and Hirshfeld surface analysis}

A significant number of weak $\mathrm{C}-\mathrm{H} \cdots \mathrm{O}, \mathrm{C}-\mathrm{H} \cdots \mathrm{N}$ and $\mathrm{N}-$ $\mathrm{H} \cdots \mathrm{O}$ intramolecular interactions and $\mathrm{C}-\mathrm{H} \cdots \mathrm{O}$ intermolecular interactions (Table 1), along with direction-specific nearly face-to-face $\pi-\pi$ stacking interactions, are responsible for the stability of the molecular packing. Intermolecular $\mathrm{C}-$ $\mathrm{H}$... O hydrogen-bond interactions forming $R_{2}^{1}(10)$ ring (Fig. 2). There are nearly face-to-face direction-specific $\pi-\pi$ stacking interactions between the centroids of the nitrophenyl ring $(x, y, z)$ and the benzene ring of the 5-bromo-2-methoxy group $(x-1, y, z)$ [centroid-centroid distance $=3.6121(5) \AA$

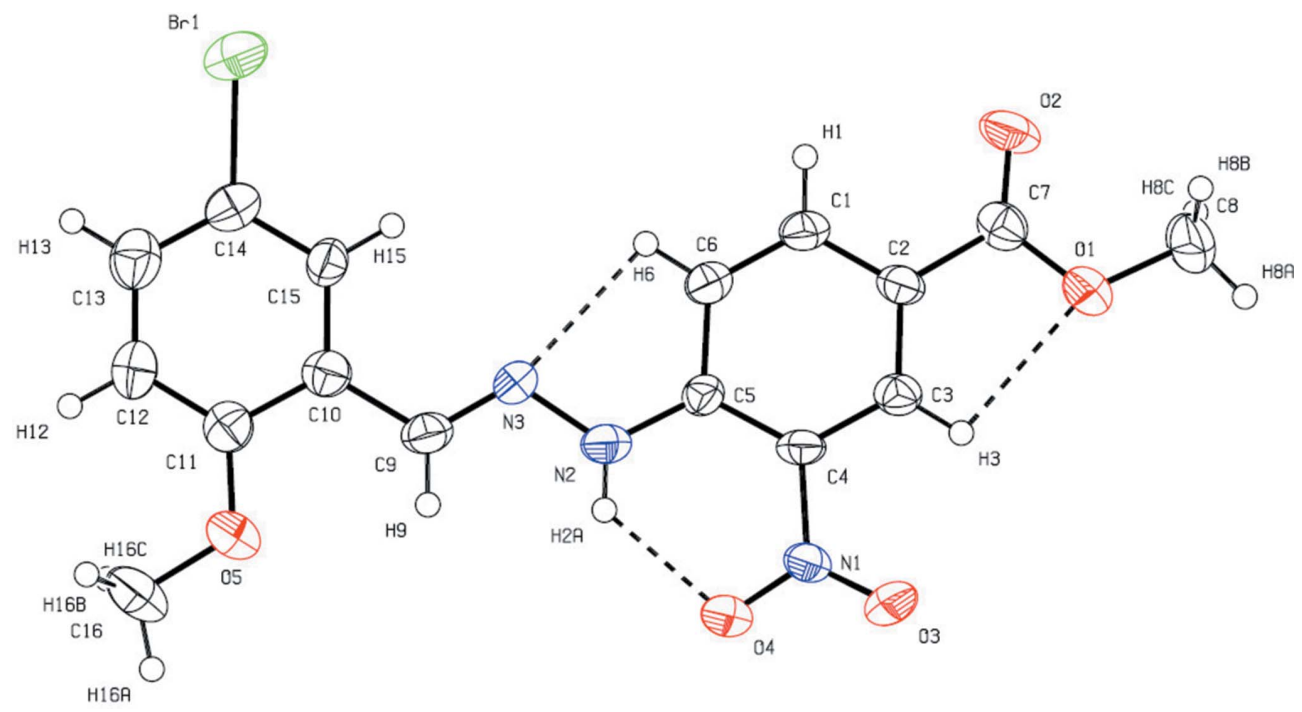

Figure 1

The molecular structure of the title compound with the atom-labelling scheme. Displacement ellipsoids are drawn at the $50 \%$ probability level. 
Table 2

Summary of the various contacts and their contributions to the Hirshfeld surface.

\begin{tabular}{ll}
\hline Contacts & Percentage contribution \\
\hline $\mathrm{Br} \cdots \mathrm{C} / \mathrm{C} \cdots \mathrm{Br}$ & 1.6 \\
$\mathrm{Br} \cdots \mathrm{H} / \mathrm{H} \cdots \mathrm{Br}$ & 11.7 \\
$\mathrm{Br} \cdots \mathrm{N} / \mathrm{N} \cdots \mathrm{Br}$ & 0.7 \\
$\mathrm{Br} \cdots \mathrm{O} / \mathrm{O} \cdots \mathrm{Br}$ & 2.8 \\
$\mathrm{C} \cdots \mathrm{C}$ & 8.1 \\
$\mathrm{C} \cdots \mathrm{H} / \mathrm{H} \cdots \mathrm{C}$ & 12.5 \\
$\mathrm{C} \cdots \mathrm{O} / \mathrm{O} \cdots \mathrm{C}$ & 2.7 \\
$\mathrm{H} \cdots \mathrm{H}$ & 27.2 \\
$\mathrm{H} \cdots \mathrm{N} / \mathrm{N} \cdots \mathrm{H}$ & 5.5 \\
$\mathrm{H} \cdots \mathrm{O} / \mathrm{O} \cdots \mathrm{H}$ & 25.1 \\
$\mathrm{~N} \cdots \mathrm{O} / \mathrm{O} \cdots \mathrm{N}$ & 1.1 \\
$\mathrm{O} \cdots \mathrm{O}$ & 1.0 \\
\hline
\end{tabular}

and slippage $=1.115 \AA$ ] , which also contributes to the molecular packing. The $\mathrm{Br}$ atom does not take part in any interactions. The nearest $\mathrm{Br} \cdots \mathrm{C} 7\left(-x+\frac{1}{2}, y-\frac{1}{2},-z+\frac{1}{2}\right)$ distance in the molecular structure is 3.6112 (7) $\AA$.

Hirshfeld surface analysis serves as a powerful tool for gaining additional insight into intermolecular interactions of molecular crystals. The Hirshfeld surfaces are mapped with 2D fingerprint plots presented using CrystalExplorer3.1 and it provides a summary of the intermolecular contacts in the crystal (McKinnon et al., 2004; Spackman \& Jayatilaka, 2009). The 2D fingerprint plots (Fig. 3) show that the intermolecular $\mathrm{H} \cdots \mathrm{H}$ and $\mathrm{O} \cdots \mathrm{H}$ interactions dominate and complement the Hirshfeld surfaces. The fingerprint plots can also be decomposed to highlight particular atom-pair close contacts (Luo et al., 2013) and enables separation of contributions from different interaction types. Two sharp spikes pointing towards the upper left of the plot are typical $\mathrm{C}-\mathrm{H} \cdots \mathrm{O}$ hydrogen bonds. This portion corresponds to $\mathrm{H} \cdots \mathrm{O}$ interactions comprising $25.1 \%$ of the total Hirshfed surfaces. Two sharp spikes pointing towards the lower left of the plot are typical $\mathrm{Br} \cdots \mathrm{H}$ hydrogen bonds. This portion corresponds to $\mathrm{Br} \cdots \mathrm{H}$ interactions comprising $11.7 \%$ of the total Hirshfeld surfaces. The broad region bearing short and narrow spikes at the middle of plot is reflected as $\mathrm{H} \cdots \mathrm{H}$ interaction comprising $27.2 \%$ of the total Hirshfeld surfaces. Apart from these, the presence of $\mathrm{Br} \cdots \mathrm{C}, \mathrm{Br} \cdots \mathrm{N}, \mathrm{Br} \cdots \mathrm{O}, \mathrm{C} \cdots \mathrm{O}, \mathrm{H} \cdots \mathrm{N}, \mathrm{N} \cdots \mathrm{O}$ and $\mathrm{O} \cdots \mathrm{O}$ interactions were observed (Pi chart; Fig. 4g), which are summarized in Table 2 (Li et al., 2013; Luo \& Sun, 2014; Seth et al., 2011).

\section{Database survey}

While searching for 2-phenylhydrazine in the Cambridge Structural Database (CSD, Version 53.7; Groom et al., 2016), four significant structures were found [CSD refcodes AYSOD (Tahir et al., 2011), DUSBID (Mufakkar et al. 2010), DUSNUB (Shad et al. 2010) and DUSNUB01 (ToledanoMagaña et al., 2015)]. Also, the crystal structure of the unsubstituted phenyl hydrazine has been reported in the CSD [ZZZGWW02 (Vickery et al., 1985) and ZZZGWW03 (Günes, et al., 2003)]. The two phenyl rings in AYSOD (two molecules in the asymmetric unit), DUSBID and DUSNUB (two molecules in the asymmetric unit) are inclined to each other by $2.44(18)$ and $14.08(19)^{\circ}$ (in molecules $A$ and $B$ ), $9.30(6)^{\circ}$, and $13.01(10)$ and $14.05(10)^{\circ}$ (in molecules $A$ and $B$ ), respectively, compared to $4.4(3)^{\circ}$ in the title compound. The crystal packing of the two compounds is significantly different. In AYSOD, $\mathrm{N}-\mathrm{H}$ groups do not form hydrogen bonds, in DUSBID, the molecules are linked by $\mathrm{N}-\mathrm{H} \cdots \pi$ interactions,

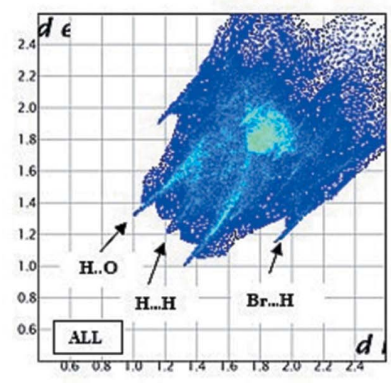

(a)

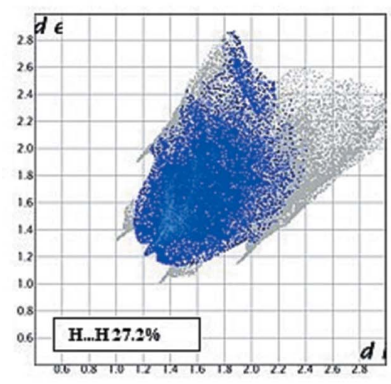

(e)

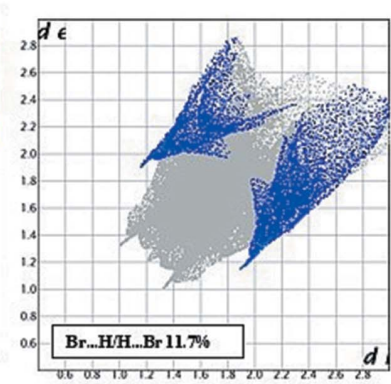

(b)

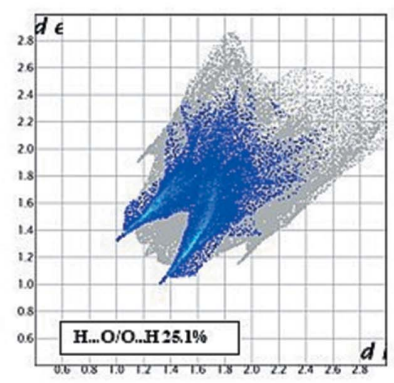

(f)

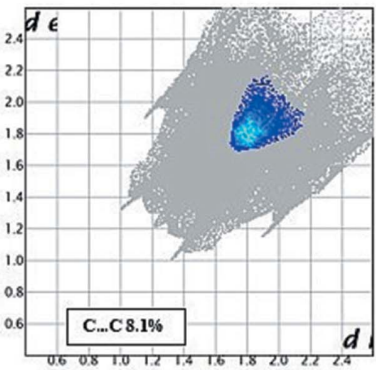

(c)

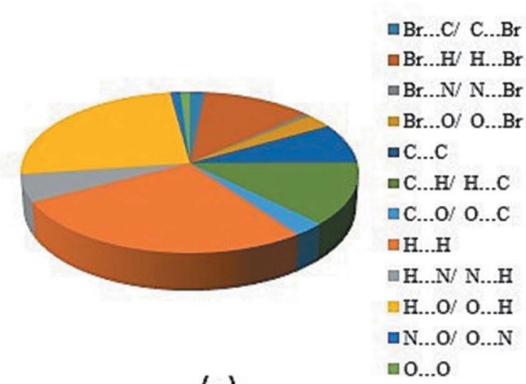

(g)

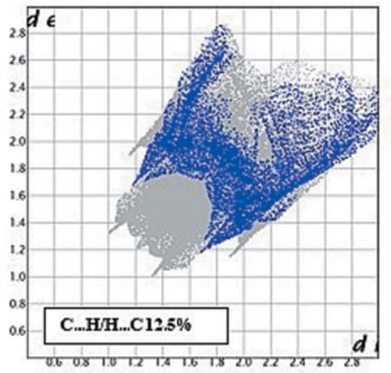

(d)

Figure 3

The full two-dimensional fingerprint plots, and those delineated into $(a)$ all interactions $(b) \mathrm{Br} \cdots \mathrm{H},(c) \mathrm{C} \cdots \mathrm{C},(d) \mathrm{C} \cdots \mathrm{H} / \mathrm{H} \cdots \mathrm{C},(e) \mathrm{H} \cdots \mathrm{H}$ and $(f)$ $\mathrm{H} \cdots \mathrm{O} / \mathrm{O} \cdots \mathrm{H}$ contacts showing the percentages of contacts contributed to the total Hirshfeld surface area. $(g)$ Pi chart. 
Table 3

Experimental details.

\begin{tabular}{|c|c|}
\hline Crystal data & \\
\hline Chemical formula & $\mathrm{C}_{16} \mathrm{H}_{14} \mathrm{BrN}_{3} \mathrm{O}_{5}$ \\
\hline$M_{\mathrm{r}}$ & 408.21 \\
\hline Crystal system, space group & Monoclinic, $P 2_{1} / n$ \\
\hline Temperature $(\mathrm{K})$ & 293 \\
\hline$a, b, c(\AA)$ & $\begin{array}{l}8.3262(11), 14.8369(19) \\
14.0764(13)\end{array}$ \\
\hline$\beta\left(^{\circ}\right)$ & $106.558(14)$ \\
\hline$V\left(\AA^{3}\right)$ & $1666.8(4)$ \\
\hline$Z$ & 4 \\
\hline Radiation type & Мо $K \alpha$ \\
\hline$\mu\left(\mathrm{mm}^{-1}\right)$ & 2.50 \\
\hline Crystal size $(\mathrm{mm})$ & $0.09 \times 0.08 \times 0.06$ \\
\hline Data collection & \\
\hline Diffractometer & Bruker APEXII CCD \\
\hline Absorption correction & Multi-scan (North et al., 1968) \\
\hline$T_{\min }, T_{\max }$ & $0.666,1.000$ \\
\hline $\begin{array}{l}\text { No. of measured, independent and } \\
\text { observed }[I>2 \sigma(I)] \text { reflections }\end{array}$ & $4830,3187,1726$ \\
\hline$R_{\text {int }}$ & 0.065 \\
\hline$(\sin \theta / \lambda)_{\max }\left(\AA^{-1}\right)$ & 0.682 \\
\hline Refinement & \\
\hline$R\left[F^{2}>2 \sigma\left(F^{2}\right)\right], w R\left(F^{2}\right), S$ & $0.096,0.202,1.09$ \\
\hline No. of reflections & 3187 \\
\hline No. of parameters & 235 \\
\hline H-atom treatment & $\begin{array}{l}\mathrm{H} \text { atoms treated by a mixture of } \\
\text { independent and constrained } \\
\text { refinement }\end{array}$ \\
\hline$\Delta \rho_{\max }, \Delta \rho_{\min }\left(\mathrm{e} \AA^{-3}\right)$ & $0.66,-0.69$ \\
\hline
\end{tabular}

Computer programs: APEX2 and SAINT (Bruker, 2008), SHELXS97 and SHELXL97 (Sheldrick, 2008) and PLATON (Spek, 2009).

and in DUSNUB, both molecules form inversion dimers linked by pairs of $\mathrm{N}-\mathrm{H} \cdots \mathrm{O}$ hydrogen bonds, thereby generating $R_{2}^{2}(16)$ motif rings (Bernstein et al., 1995). In the title compound, intramolecular $\mathrm{N}-\mathrm{H} \cdots \mathrm{O}$ and only intermolecular $\mathrm{C}-\mathrm{H} \cdots \mathrm{O}$ hydrogen bonds are present; there are no $\mathrm{C}-\mathrm{H} \cdots \pi$ interactions. Very few similar hydrazine derivatives are reported in the literature (Cortés et al., 2013; Dey \& Chopra, 2017). In those crystal structures, a halogen group ( $\mathrm{Cl}$ and $\mathrm{F}$, respectively) is present, while in this crystal structure, $\mathrm{Br}$ is present.

\section{Synthesis and crystallization}

The title compound was synthesized in one step by heating the hydrazine derivative 3 -nitrobenzohydrazide $(0.181 \mathrm{mg})$ with a slight excess of 5-bromo-2-methoxybenzaldehyde $(0.215 \mathrm{mg})$ in an acetic acid solution $(10 \mathrm{ml})$. The reaction mixture was refluxed for $8 \mathrm{~h}$. The solid product formed during reflux was filtered off, washed and dried over anhydrous calcium chloride in a vacuum desiccator (yield $75 \%$ ). The final product was soluble in acetone, dimethyl sulfoxide (DMSO), dimethylformamide (DMF), methanol, ethanol and ethyl acetate, etc. Transparent orange-coloured needle-shaped diffractionquality single crystals of the title compound were grown by slow evaporation using methanol as the solvent at room temperature.

\section{Refinement}

Crystal data, data collection and structure refinement details are summarized in Table 3. The coordinates of the $\mathrm{H}$ atoms of the $\mathrm{N} 2-\mathrm{H} 2$ and $\mathrm{C} 9-\mathrm{H} 9$ groups were refined $[\mathrm{N} 2-\mathrm{H} 2=$ 0.83 (6) $\AA$ and $\mathrm{C} 9-\mathrm{H} 9=0.90(5) \AA]$. Other $\mathrm{H}$ atoms were placed in geometrically idealized positions and constrained to ride on their parent atoms, with $\mathrm{C}-\mathrm{H}=0.93-0.97 \AA$, and refined as riding with $U_{\text {iso }}(\mathrm{H})=x U_{\text {eq }}(\mathrm{C})$, where $x=1.5$ for methyl and $x=1.2$ for all other $\mathrm{H}$ atoms.

\section{Acknowledgements}

Authors are thankful to the DST-FIST, New Delhi, for providing the Kappa APEXII single-crystal X-ray diffractometer facility at Department of Physics, Sardar Patel University, Vallabh vidyanagar, Gujarat, India. One of the authors (TJM) is thankful to LDRP-Institute of Technology \& Research, Gandhinagar, for giving necessary permission.

\section{References}

Banerjee, S., Mondal, S., Chakraborty, W., Sen, S., Gachhui, R., Butcher, R. J., Slawin, A. M. Z., Mandal, C. \& Mitra, S. (2009). Polyhedron, 28, 2785-2793.

Belskaya, N. P., Dehaen, W. \& Bakulev, V. A. (2010). Arch. Org. Chem. 1, 275-332.

Bernstein, J., Davis, R. E., Shimoni, L. \& Chang, N.-L. (1995). Angew. Chem. Int. Ed. Engl. 34, 1555-1573.

Bruker (2008). APEX2 and SAINT. Bruker AXS Inc., Madison, Wisconsin, USA.

Corey, E. J. \& Enders, D. (1976). Tetrahedron Lett. 17, 11-14.

Cortés, E., Abonía, R., Cobo, J. \& Glidewell, C. (2013). Acta Cryst. C69, 754-760.

Dey, D. \& Chopra, D. (2017). Acta Cryst. B73, 781-793.

Groom, C. R., Bruno, I. J., Lightfoot, M. P. \& Ward, S. C. (2016). Acta Cryst. B72, 171-179.

Günes, B., Özbey, S. \& Tezcan, H. (2003). Anal. Sci. 19, 1091-1092.

Levrand, B., Fiebera, W., Lehn, J.-M. \& Herrmann, A. (2007). Helv. Chim. Acta, 90, 2281-2314.

Li, Y., Zhang, C. G., Cai, L. Y. \& Wang, Z. X. (2013). J. Coord. Chem. 66, 3100-3112.

Li, L., Zhu, L., Chen, D., Hu, X. \& Wang, R. (2011). Eur. J. Org. Chem. pp. 2692-2696.

Luo, Y. H. \& Sun, B. W. (2014). Spectrochim. Acta Part A, 120, 381388.

Luo, Y. H., Wu, G. G., Mao, S. L. \& Sun, B. W. (2013). Inorg. Chim. Acta, 397, 1-9.

McKinnon, J. J., Spackman, M. A. \& Mitchell, A. S. (2004). Acta Cryst. B60, 627-668.

Mufakkar, M., Tahir, M. N., Tariq, M. I., Ahmad, S. \& Sarfraz, M. (2010). Acta Cryst. E66, o1887.

Narang, R., Narasimhan, B. \& Sharma, S. (2012). Curr. Med. Chem. 19, 569-612.

North, A. C. T., Phillips, D. C. \& Mathews, F. S. (1968). Acta Cryst. A24, 351-359.

Rollas, S. \& Küçükgüzel, S. G. (2007). Molecules, 12, 1910-1939.

Seth, S. K., Mandal, P. C., Kar, T. \& Mukhopadhyay, S. (2011). J. Mol. Struct. 994, 109-116.

Shad, H. A., Tahir, M. N., Tariq, M. I., Sarfraz, M. \& Ahmad, S. (2010). Acta Cryst. E66, o1955.

Sheldrick, G. M. (2008). Acta Cryst. A64, 112-122.

Spackman, M. A. \& Jayatilaka, D. (2009). CrystEngComm, 11, 19-32. Spek, A. L. (2009). Acta Cryst. D65, 148-155.

Tahir, M. N., Tariq, M. I., Tariq, R. H. \& Sarfraz, M. (2011). Acta Cryst. E67, o2377. 
Toledano-Magaña, Y., García-Ramos, J. C., Navarro-Olivarria, M., Flores-Alamo, M., Manzanera-Estrada, M., Ortiz-Frade, L., Galindo-Murillo, R., Ruiz-Azuara, L., Meléndrez-Luevano, R. M. \& Cabrera-Vivas, B. M. (2015). Molecules, 20, 9929-9948.

Uppal, G., Bala, S., Kamboj, S. \& Saini, M. (2011). Der. Pharma Chem. 3, 250-68.
Vickery, B., Willey, G. R. \& Drew, M. G. B. (1985). Acta Cryst. C41, 1072-1075.

Wu, A., Senter, M. \& Peter, D. (2005). Nat. Biotechnol. 23, 1137-1146. Xavier, A. J., Thakur, M. \& Marie, J. M. (2012). J. Chem. Pharm. Res. 4, 986-990. 


\title{
supporting information
}

Acta Cryst. (2018). E74, 1239-1243 [https://doi.org/10.1107/S2056989018011325]

\section{Crystal structure and Hirshfeld surface analysis of methyl 4-[(E)-2-(5-bromo-2- methoxybenzylidene)hydrazinyl]-3-nitrobenzoate}

\author{
Tanvirbanu J. Malek, Sahaj A. Gandhi, Vijay Barot, Mukesh Patel and Urmila H. Patel \\ Computing details
}

Data collection: APEX2 (Bruker, 2008); cell refinement: SAINT (Bruker, 2008); data reduction: SAINT (Bruker, 2008); program(s) used to solve structure: SHELXS97 (Sheldrick, 2008); program(s) used to refine structure: SHELXL97 (Sheldrick, 2008); molecular graphics: PLATON (Spek, 2009); software used to prepare material for publication: SHELXL97 (Sheldrick, 2008).

Methyl 4-[(E)-2-(5-bromo-2-methoxybenzylidene)hydrazinyl]-3-nitrobenzoate

Crystal data

$\mathrm{C}_{16} \mathrm{H}_{14} \mathrm{BrN}_{3} \mathrm{O}_{5}$

$M_{r}=408.21$

Monoclinic, $P 2{ }_{1} / n$

$a=8.3262(11) \AA$

$b=14.8369(19) \AA$

$c=14.0764(13) \AA$

$\beta=106.558(14)^{\circ}$

$V=1666.8(4) \AA^{3}$

$Z=4$

\section{Data collection}

Bruker APEXII CCD diffractometer

Radiation source: sealed tube $\varphi$ and $\omega$ scans

Absorption correction: multi-scan (North et al., 1968)

$T_{\min }=0.666, T_{\max }=1.000$

4830 measured reflections

\section{Refinement}

Refinement on $F^{2}$

Least-squares matrix: full

$R\left[F^{2}>2 \sigma\left(F^{2}\right)\right]=0.096$

$w R\left(F^{2}\right)=0.202$

$S=1.09$

3187 reflections

235 parameters

0 restraints

Hydrogen site location: mixed
$F(000)=824$

$D_{\mathrm{x}}=1.627 \mathrm{Mg} \mathrm{m}^{-3}$

Mo $K \alpha$ radiation, $\lambda=0.71073 \AA$

Cell parameters from 1261 reflections

$\theta=3.3-23.2^{\circ}$

$\mu=2.50 \mathrm{~mm}^{-1}$

$T=293 \mathrm{~K}$

Plate, yellow

$0.09 \times 0.08 \times 0.06 \mathrm{~mm}$

3187 independent reflections

1726 reflections with $I>2 \sigma(I)$

$R_{\text {int }}=0.065$

$\theta_{\max }=29.0^{\circ}, \theta_{\min }=3.6^{\circ}$

$h=-10 \rightarrow 11$

$k=-19 \rightarrow 10$

$l=-9 \rightarrow 18$

$\mathrm{H}$ atoms treated by a mixture of independent and constrained refinement

$w=1 /\left[\sigma^{2}\left(F_{\mathrm{o}}^{2}\right)+(0.0381 P)^{2}+5.1653 P\right]$ where $P=\left(F_{\mathrm{o}}^{2}+2 F_{\mathrm{c}}{ }^{2}\right) / 3$

$(\Delta / \sigma)_{\max }<0.001$

$\Delta \rho_{\max }=0.66 \mathrm{e} \AA^{-3}$

$\Delta \rho_{\min }=-0.69$ e $\AA^{-3}$

Extinction correction: SHELXL97 (Sheldrick, 2008), $\mathrm{Fc}^{*}=\mathrm{kFc}\left[1+0.001 \mathrm{xFc}^{2} \lambda^{3} / \sin (2 \theta)\right]^{-1 / 4}$

Extinction coefficient: 0.0076 (10) 


\section{Special details}

Geometry. All esds (except the esd in the dihedral angle between two 1.s. planes) are estimated using the full covariance matrix. The cell esds are taken into account individually in the estimation of esds in distances, angles and torsion angles; correlations between esds in cell parameters are only used when they are defined by crystal symmetry. An approximate (isotropic) treatment of cell esds is used for estimating esds involving l.s. planes.

Fractional atomic coordinates and isotropic or equivalent isotropic displacement parameters $\left(\AA^{2}\right)$

\begin{tabular}{|c|c|c|c|c|}
\hline & $x$ & $y$ & $z$ & $U_{\text {iso }} * / U_{\text {eq }}$ \\
\hline $\mathrm{C} 1$ & $0.3935(9)$ & $0.2162(5)$ & $0.6270(5)$ & $0.038(2)$ \\
\hline H1 & 0.415174 & 0.223200 & 0.695123 & $0.046^{*}$ \\
\hline $\mathrm{C} 2$ & $0.2457(9)$ & $0.1749(5)$ & $0.5739(5)$ & 0.0348 (19) \\
\hline $\mathrm{C} 3$ & $0.2169(9)$ & $0.1645(5)$ & $0.4728(5)$ & $0.0339(18)$ \\
\hline H3 & 0.118584 & 0.137270 & 0.435409 & $0.041^{*}$ \\
\hline $\mathrm{C} 4$ & $0.3340(9)$ & $0.1944(5)$ & $0.4272(5)$ & $0.0328(18)$ \\
\hline $\mathrm{C} 5$ & $0.4810(9)$ & $0.2391(5)$ & $0.4784(5)$ & $0.0308(17)$ \\
\hline C6 & $0.5083(9)$ & $0.2468(6)$ & $0.5818(5)$ & $0.040(2)$ \\
\hline H6 & 0.606744 & 0.273342 & 0.620051 & $0.049^{*}$ \\
\hline $\mathrm{C} 7$ & $0.1197(10)$ & $0.1486(6)$ & $0.6245(6)$ & $0.0386(19)$ \\
\hline $\mathrm{C} 8$ & $-0.1484(10)$ & $0.0834(7)$ & $0.6023(6)$ & $0.061(3)$ \\
\hline H8A & -0.236862 & 0.055874 & 0.551367 & $0.091 *$ \\
\hline H8B & -0.107222 & 0.041302 & 0.655468 & $0.091 *$ \\
\hline $\mathrm{H} 8 \mathrm{C}$ & -0.190635 & 0.135968 & 0.626904 & $0.091 *$ \\
\hline C9 & $0.8528(10)$ & $0.3310(6)$ & $0.4527(5)$ & $0.040(2)$ \\
\hline $\mathrm{C} 10$ & $1.0108(9)$ & $0.3711(6)$ & $0.5111(5)$ & $0.0373(19)$ \\
\hline C11 & $1.1267(10)$ & $0.4052(5)$ & $0.4648(6)$ & $0.040(2)$ \\
\hline $\mathrm{C} 12$ & $1.2709(10)$ & $0.4470(6)$ & $0.5203(6)$ & $0.046(2)$ \\
\hline H12 & 1.345065 & 0.471252 & 0.488742 & $0.055^{*}$ \\
\hline $\mathrm{C} 13$ & $1.3069(10)$ & $0.4532(6)$ & $0.6226(6)$ & $0.052(2)$ \\
\hline H13 & 1.404718 & 0.480982 & 0.659877 & $0.063^{*}$ \\
\hline $\mathrm{C} 14$ & $1.1940(10)$ & $0.4174(6)$ & $0.6681(5)$ & $0.043(2)$ \\
\hline $\mathrm{C} 15$ & $1.0485(9)$ & $0.3762(5)$ & $0.6140(5)$ & $0.0357(18)$ \\
\hline H15 & 0.975310 & 0.351682 & 0.646202 & $0.043^{*}$ \\
\hline $\mathrm{C} 16$ & $1.1856(12)$ & $0.4401(7)$ & $0.3120(6)$ & 0.069 (3) \\
\hline H16A & 1.141419 & 0.428322 & 0.242387 & $0.104^{*}$ \\
\hline H16B & 1.185656 & 0.503869 & 0.323555 & $0.104^{*}$ \\
\hline $\mathrm{H} 16 \mathrm{C}$ & 1.298062 & 0.417511 & 0.335016 & $0.104^{*}$ \\
\hline N1 & $0.2911(8)$ & $0.1797(5)$ & $0.3208(4)$ & $0.0370(16)$ \\
\hline $\mathrm{N} 2$ & $0.5978(8)$ & $0.2714(5)$ & $0.4364(5)$ & $0.0424(18)$ \\
\hline N3 & $0.7457(8)$ & $0.3049(5)$ & 0.4962 (4) & $0.0393(16)$ \\
\hline $\mathrm{O} 1$ & $-0.0146(7)$ & 0.1088 (4) & $0.5621(4)$ & $0.0494(16)$ \\
\hline $\mathrm{O} 2$ & $0.1325(7)$ & $0.1613(4)$ & 0.7107 (4) & $0.0548(17)$ \\
\hline $\mathrm{O} 3$ & $0.1809(8)$ & $0.1260(5)$ & 0.2817 (4) & $0.0612(18)$ \\
\hline $\mathrm{O} 4$ & $0.3693(6)$ & 0.2228 (4) & $0.2728(3)$ & $0.0544(17)$ \\
\hline O5 & $1.0838(7)$ & $0.3963(4)$ & $0.3644(4)$ & $0.0545(17)$ \\
\hline BR1 & $1.24638(13)$ & $0.41918(8)$ & $0.80847(6)$ & $0.0681(5)$ \\
\hline $\mathrm{H} 2 \mathrm{~A}$ & $0.576(7)$ & $0.270(4)$ & $0.375(4)$ & $0.014(16)^{*}$ \\
\hline H9 & $0.825(7)$ & $0.329(4)$ & $0.386(4)$ & $0.012(15)^{*}$ \\
\hline
\end{tabular}


Atomic displacement parameters $\left(\AA^{2}\right)$

\begin{tabular}{lllllll}
\hline & $U^{11}$ & $U^{22}$ & $U^{33}$ & $U^{12}$ & $U^{13}$ & $U^{23}$ \\
\hline C1 & $0.037(4)$ & $0.050(6)$ & $0.025(3)$ & $0.001(4)$ & $0.004(4)$ & $0.002(4)$ \\
C2 & $0.035(4)$ & $0.046(5)$ & $0.025(3)$ & $0.004(4)$ & $0.011(3)$ & $0.004(3)$ \\
C3 & $0.033(4)$ & $0.040(5)$ & $0.028(3)$ & $0.005(4)$ & $0.007(3)$ & $0.004(3)$ \\
C4 & $0.038(4)$ & $0.040(5)$ & $0.020(3)$ & $0.002(4)$ & $0.008(3)$ & $-0.003(3)$ \\
C5 & $0.025(4)$ & $0.039(5)$ & $0.028(3)$ & $0.001(4)$ & $0.006(3)$ & $-0.003(3)$ \\
C6 & $0.033(4)$ & $0.056(6)$ & $0.030(3)$ & $-0.003(4)$ & $0.006(4)$ & $-0.005(4)$ \\
C7 & $0.038(4)$ & $0.041(6)$ & $0.039(4)$ & $0.008(4)$ & $0.016(4)$ & $0.004(4)$ \\
C8 & $0.038(5)$ & $0.091(8)$ & $0.059(5)$ & $-0.003(5)$ & $0.022(4)$ & $0.007(5)$ \\
C9 & $0.037(5)$ & $0.051(6)$ & $0.027(4)$ & $0.002(4)$ & $0.004(4)$ & $-0.001(4)$ \\
C10 & $0.032(4)$ & $0.044(5)$ & $0.035(4)$ & $0.007(4)$ & $0.008(4)$ & $0.003(4)$ \\
C11 & $0.035(4)$ & $0.040(6)$ & $0.042(4)$ & $0.006(4)$ & $0.010(4)$ & $0.004(4)$ \\
C12 & $0.039(5)$ & $0.042(6)$ & $0.056(5)$ & $-0.008(4)$ & $0.012(4)$ & $-0.001(4)$ \\
C13 & $0.039(5)$ & $0.055(6)$ & $0.060(5)$ & $0.000(4)$ & $0.011(5)$ & $0.003(5)$ \\
C14 & $0.044(5)$ & $0.042(6)$ & $0.037(4)$ & $0.003(4)$ & $0.002(4)$ & $-0.001(4)$ \\
C15 & $0.031(4)$ & $0.037(5)$ & $0.038(4)$ & $-0.006(4)$ & $0.007(4)$ & $-0.003(4)$ \\
C16 & $0.073(6)$ & $0.095(9)$ & $0.052(5)$ & $-0.006(6)$ & $0.037(5)$ & $0.010(5)$ \\
N1 & $0.034(4)$ & $0.053(5)$ & $0.025(3)$ & $0.001(3)$ & $0.011(3)$ & $0.003(3)$ \\
N2 & $0.038(4)$ & $0.064(5)$ & $0.025(3)$ & $-0.002(4)$ & $0.008(3)$ & $-0.004(3)$ \\
N3 & $0.033(4)$ & $0.053(5)$ & $0.030(3)$ & $-0.009(3)$ & $0.004(3)$ & $-0.003(3)$ \\
O1 & $0.044(3)$ & $0.070(5)$ & $0.040(3)$ & $-0.005(3)$ & $0.021(3)$ & $0.000(3)$ \\
O2 & $0.060(4)$ & $0.079(5)$ & $0.031(3)$ & $0.002(3)$ & $0.023(3)$ & $0.000(3)$ \\
O3 & $0.065(4)$ & $0.081(5)$ & $0.032(3)$ & $-0.035(4)$ & $0.004(3)$ & $-0.012(3)$ \\
O4 & $0.046(3)$ & $0.090(5)$ & $0.028(3)$ & $-0.012(3)$ & $0.013(3)$ & $-0.001(3)$ \\
O5 & $0.054(4)$ & $0.075(5)$ & $0.038(3)$ & $-0.008(3)$ & $0.020(3)$ & $0.005(3)$ \\
BR1 & $0.0721(8)$ & $0.0855(9)$ & $0.0375(5)$ & $-0.0200(6)$ & $0.0007(5)$ & $-0.0053(5)$ \\
& & & & & & \\
\hline & & & & & & \\
\end{tabular}

Geometric parameters $\left(\AA,{ }^{\circ}\right)$

\begin{tabular}{llll}
\hline $\mathrm{C} 1-\mathrm{C} 6$ & $1.369(10)$ & $\mathrm{C} 9-\mathrm{H} 9$ & $0.90(5)$ \\
$\mathrm{C} 1-\mathrm{C} 2$ & $1.386(10)$ & $\mathrm{C} 10-\mathrm{C} 15$ & $1.394(9)$ \\
$\mathrm{C} 1-\mathrm{H} 1$ & 0.9300 & $\mathrm{C} 10-\mathrm{C} 11$ & $1.404(10)$ \\
$\mathrm{C} 2-\mathrm{C} 3$ & $1.383(9)$ & $\mathrm{C} 11-\mathrm{O} 5$ & $1.362(9)$ \\
$\mathrm{C} 2-\mathrm{C} 7$ & $1.479(10)$ & $\mathrm{C} 11-\mathrm{C} 12$ & $1.379(11)$ \\
$\mathrm{C} 3-\mathrm{C} 4$ & $1.385(9)$ & $\mathrm{C} 12-\mathrm{C} 13$ & $1.388(11)$ \\
$\mathrm{C} 3-\mathrm{H} 3$ & 0.9300 & $\mathrm{C} 12-\mathrm{H} 12$ & 0.9300 \\
$\mathrm{C} 4-\mathrm{C} 5$ & $1.399(10)$ & $\mathrm{C} 13-\mathrm{C} 14$ & $1.385(11)$ \\
$\mathrm{C} 4-\mathrm{N} 1$ & $1.454(8)$ & $\mathrm{C} 13-\mathrm{H} 13$ & 0.9300 \\
$\mathrm{C} 5-\mathrm{N} 2$ & $1.361(9)$ & $\mathrm{C} 14-\mathrm{C} 15$ & $1.377(10)$ \\
$\mathrm{C} 5-\mathrm{C} 6$ & $1.411(9)$ & $\mathrm{C} 14-\mathrm{BR} 1$ & $1.899(7)$ \\
$\mathrm{C} 6-\mathrm{H} 6$ & 0.9300 & $\mathrm{C} 15-\mathrm{H} 15$ & 0.9300 \\
$\mathrm{C} 7-\mathrm{O} 2$ & $1.201(8)$ & $\mathrm{C} 16-\mathrm{O} 5$ & $1.428(9)$ \\
$\mathrm{C} 7-\mathrm{O} 1$ & $1.346(9)$ & $\mathrm{C} 16-\mathrm{H} 16 \mathrm{~A}$ & 0.9600 \\
$\mathrm{C} 8-\mathrm{O} 1$ & $1.437(9)$ & $\mathrm{C} 16-\mathrm{H} 16 \mathrm{~B}$ & 0.9600 \\
$\mathrm{C} 8-\mathrm{H} 8 \mathrm{~A}$ & 0.9600 & $\mathrm{C} 16-\mathrm{H} 16 \mathrm{C}$ & 0.9600 \\
$\mathrm{C} 8-\mathrm{H} 8 \mathrm{~B}$ & 0.9600 & $\mathrm{~N} 1-\mathrm{O} 3$ & $1.221(8)$
\end{tabular}




\begin{tabular}{|c|c|c|c|}
\hline $\mathrm{C} 8-\mathrm{H} 8 \mathrm{C}$ & 0.9600 & $\mathrm{~N} 1-\mathrm{O} 4$ & $1.240(7)$ \\
\hline $\mathrm{C} 9-\mathrm{N} 3$ & $1.277(9)$ & $\mathrm{N} 2-\mathrm{N} 3$ & $1.371(8)$ \\
\hline $\mathrm{C} 9-\mathrm{C} 10$ & $1.464(11)$ & $\mathrm{N} 2-\mathrm{H} 2 \mathrm{~A}$ & $0.83(6)$ \\
\hline $\mathrm{C} 6-\mathrm{C} 1-\mathrm{C} 2$ & $121.8(6)$ & $\mathrm{C} 11-\mathrm{C} 10-\mathrm{C} 9$ & $120.8(7)$ \\
\hline $\mathrm{C} 6-\mathrm{C} 1-\mathrm{H} 1$ & 119.1 & $\mathrm{O} 5-\mathrm{C} 11-\mathrm{C} 12$ & $124.1(7)$ \\
\hline $\mathrm{C} 2-\mathrm{C} 1-\mathrm{H} 1$ & 119.1 & $\mathrm{O} 5-\mathrm{C} 11-\mathrm{C} 10$ & $115.8(7)$ \\
\hline $\mathrm{C} 3-\mathrm{C} 2-\mathrm{C} 1$ & $118.1(7)$ & $\mathrm{C} 12-\mathrm{C} 11-\mathrm{C} 10$ & $120.1(7)$ \\
\hline $\mathrm{C} 3-\mathrm{C} 2-\mathrm{C} 7$ & $121.8(7)$ & $\mathrm{C} 11-\mathrm{C} 12-\mathrm{C} 13$ & $120.9(8)$ \\
\hline $\mathrm{C} 1-\mathrm{C} 2-\mathrm{C} 7$ & $120.0(6)$ & $\mathrm{C} 11-\mathrm{C} 12-\mathrm{H} 12$ & 119.5 \\
\hline $\mathrm{C} 2-\mathrm{C} 3-\mathrm{C} 4$ & $120.2(7)$ & $\mathrm{C} 13-\mathrm{C} 12-\mathrm{H} 12$ & 119.5 \\
\hline $\mathrm{C} 2-\mathrm{C} 3-\mathrm{H} 3$ & 119.9 & $\mathrm{C} 14-\mathrm{C} 13-\mathrm{C} 12$ & $118.6(8)$ \\
\hline $\mathrm{C} 4-\mathrm{C} 3-\mathrm{H} 3$ & 119.9 & $\mathrm{C} 14-\mathrm{C} 13-\mathrm{H} 13$ & 120.7 \\
\hline $\mathrm{C} 3-\mathrm{C} 4-\mathrm{C} 5$ & $122.8(6)$ & $\mathrm{C} 12-\mathrm{C} 13-\mathrm{H} 13$ & 120.7 \\
\hline $\mathrm{C} 3-\mathrm{C} 4-\mathrm{N} 1$ & $115.5(7)$ & $\mathrm{C} 15-\mathrm{C} 14-\mathrm{C} 13$ & $121.5(7)$ \\
\hline $\mathrm{C} 5-\mathrm{C} 4-\mathrm{N} 1$ & $121.7(6)$ & $\mathrm{C} 15-\mathrm{C} 14-\mathrm{BR} 1$ & $119.1(6)$ \\
\hline $\mathrm{N} 2-\mathrm{C} 5-\mathrm{C} 4$ & $124.8(6)$ & $\mathrm{C} 13-\mathrm{C} 14-\mathrm{BR} 1$ & $119.4(6)$ \\
\hline $\mathrm{N} 2-\mathrm{C} 5-\mathrm{C} 6$ & $119.6(7)$ & $\mathrm{C} 14-\mathrm{C} 15-\mathrm{C} 10$ & $120.0(7)$ \\
\hline $\mathrm{C} 4-\mathrm{C} 5-\mathrm{C} 6$ & $115.5(6)$ & $\mathrm{C} 14-\mathrm{C} 15-\mathrm{H} 15$ & 120.0 \\
\hline $\mathrm{C} 1-\mathrm{C} 6-\mathrm{C} 5$ & $121.6(7)$ & $\mathrm{C} 10-\mathrm{C} 15-\mathrm{H} 15$ & 120.0 \\
\hline $\mathrm{C} 1-\mathrm{C} 6-\mathrm{H} 6$ & 119.2 & $\mathrm{O} 5-\mathrm{C} 16-\mathrm{H} 16 \mathrm{~A}$ & 109.5 \\
\hline $\mathrm{C} 5-\mathrm{C} 6-\mathrm{H} 6$ & 119.2 & $\mathrm{O} 5-\mathrm{C} 16-\mathrm{H} 16 \mathrm{~B}$ & 109.5 \\
\hline $\mathrm{O} 2-\mathrm{C} 7-\mathrm{O} 1$ & $123.1(7)$ & $\mathrm{H} 16 \mathrm{~A}-\mathrm{C} 16-\mathrm{H} 16 \mathrm{~B}$ & 109.5 \\
\hline $\mathrm{O} 2-\mathrm{C} 7-\mathrm{C} 2$ & $125.0(8)$ & $\mathrm{O} 5-\mathrm{C} 16-\mathrm{H} 16 \mathrm{C}$ & 109.5 \\
\hline $\mathrm{O} 1-\mathrm{C} 7-\mathrm{C} 2$ & $111.9(6)$ & $\mathrm{H} 16 \mathrm{~A}-\mathrm{C} 16-\mathrm{H} 16 \mathrm{C}$ & 109.5 \\
\hline $\mathrm{O} 1-\mathrm{C} 8-\mathrm{H} 8 \mathrm{~A}$ & 109.5 & $\mathrm{H} 16 \mathrm{~B}-\mathrm{C} 16-\mathrm{H} 16 \mathrm{C}$ & 109.5 \\
\hline $\mathrm{O} 1-\mathrm{C} 8-\mathrm{H} 8 \mathrm{~B}$ & 109.5 & $\mathrm{O} 3-\mathrm{N} 1-\mathrm{O} 4$ & $122.3(6)$ \\
\hline $\mathrm{H} 8 \mathrm{~A}-\mathrm{C} 8-\mathrm{H} 8 \mathrm{~B}$ & 109.5 & $\mathrm{O} 3-\mathrm{N} 1-\mathrm{C} 4$ & $119.6(6)$ \\
\hline $\mathrm{O} 1-\mathrm{C} 8-\mathrm{H} 8 \mathrm{C}$ & 109.5 & $\mathrm{O} 4-\mathrm{N} 1-\mathrm{C} 4$ & $118.0(6)$ \\
\hline $\mathrm{H} 8 \mathrm{~A}-\mathrm{C} 8-\mathrm{H} 8 \mathrm{C}$ & 109.5 & $\mathrm{C} 5-\mathrm{N} 2-\mathrm{N} 3$ & $119.2(6)$ \\
\hline $\mathrm{H} 8 \mathrm{~B}-\mathrm{C} 8-\mathrm{H} 8 \mathrm{C}$ & 109.5 & $\mathrm{C} 5-\mathrm{N} 2-\mathrm{H} 2 \mathrm{~A}$ & $118(4)$ \\
\hline $\mathrm{N} 3-\mathrm{C} 9-\mathrm{C} 10$ & $119.5(7)$ & $\mathrm{N} 3-\mathrm{N} 2-\mathrm{H} 2 \mathrm{~A}$ & $122(4)$ \\
\hline N3-C9-H9 & $119(4)$ & $\mathrm{C} 9-\mathrm{N} 3-\mathrm{N} 2$ & $116.3(6)$ \\
\hline $\mathrm{C} 10-\mathrm{C} 9-\mathrm{H} 9$ & $121(4)$ & $\mathrm{C} 7-\mathrm{O} 1-\mathrm{C} 8$ & $116.8(6)$ \\
\hline $\mathrm{C} 15-\mathrm{C} 10-\mathrm{C} 11$ & $118.9(7)$ & $\mathrm{C} 11-\mathrm{O} 5-\mathrm{C} 16$ & $118.2(7)$ \\
\hline $\mathrm{C} 15-\mathrm{C} 10-\mathrm{C} 9$ & $120.3(7)$ & & \\
\hline
\end{tabular}

Hydrogen-bond geometry $\left(\AA,{ }^{\circ}\right)$

\begin{tabular}{lllll}
\hline$D-\mathrm{H} \cdots A$ & $D-\mathrm{H}$ & $\mathrm{H} \cdots A$ & $D \cdots A$ & $D-\mathrm{H} \cdots A$ \\
\hline $\mathrm{N} 2-\mathrm{H} 2 A \cdots \mathrm{O} 4$ & 0.83 & 2.03 & $2.635(3)$ & 129 \\
$\mathrm{C} 3-\mathrm{H} 3 \cdots \mathrm{O} 1$ & 0.93 & 2.39 & $2.712(4)$ & 100 \\
$\mathrm{C} 6-\mathrm{H} 6 \cdots \mathrm{N} 3$ & 0.93 & 2.40 & $2.731(4)$ & 101 \\
$\mathrm{C} 6-\mathrm{H} 6 \cdots \mathrm{O} 4$ & 0.93 & 2.59 & $3.444(5)$ & 152 \\
$\mathrm{C} 15-\mathrm{H} 15 \cdots \mathrm{O} 4{ }^{\mathrm{i}}$ & 0.93 & 2.46 & $3.358(4)$ & 161 \\
\hline
\end{tabular}

Symmetry code: (i) $x-1 / 2,-y-1 / 2, z-1 / 2$. 